\title{
Associative Binary Operations on a Set with Five Elements
}

\author{
Amit Sehgal \\ Mathematics Department, \\ Govt. College, Birohar (Jhajjar) \\ (Haryana), India
}

\author{
Ravinder Kumar \\ Mathematics Department, \\ A.I.J.H.M. College, Rohtak \\ (Haryana), India
}

\author{
Ombir Dahiya \\ Mathematics Department, \\ A.I.J.H.M. College, Rohtak \\ (Haryana), India
}

\begin{abstract}
Associativity of a binary operation is one of most fundamental property in algebra. The number of binary operation on a set of only five elements is as large as $298,023,223,876,953,125$. In this paper, the authors give the answer of the question "How many binary operations on a five elements are associative?" The main goal of this paper is to extend [3] and [5] for five element set.
\end{abstract}

\section{Keywords}

binary operations, associativity, isomorphism, order, Partition

\section{INTRODUCTION}

The number of binary operation on a set of only five elements is as large as $298,023,223,876,953,125$. To prove this is an easy calculation; there are five different answers for each of the 25 seats of a $5 \times 5$ operation table so the number of distinct binary operations is $5^{25}$.This calculation is straightforward, but no easy calculation and no educated guess, seems to give the answer to following question:-

"How many binary operations on a five elements are associative?"

The objective of this paper is to answer this question. In other words, how many of the $298,023,223,876,953,125$ different binary operations on five-element set are associative. The main goal of this paper is to extend [3] and [5] for five element set.

\section{GENERAL CONCEPTS}

A binary operation (hereafter referred to only as an operation) on a set $\mathrm{S}$ is a rule that assigns to each ordered pair $(\mathrm{a}, \mathrm{b})$, where $\mathrm{a}$ and $\mathrm{b}$ are elements of $\mathrm{S}$, exactly one element, denoted by $a b$, in $S$.

An operation on a set is associative if $\mathrm{x}(\mathrm{yz})=(\mathrm{xy}) \mathrm{z}$ for every $\mathrm{x}, \mathrm{y}$ and $\mathrm{z}$ in $\mathrm{S}$.

An isomorphism between $\mathrm{S}$ and $\mathrm{S}$ ' is a one-to-one function $\Phi$ mapping S onto S' such that $\Phi(\mathrm{xy})=\Phi(\mathrm{x}) \Phi(\mathrm{y})$ for all $\mathrm{x}$ and $\mathrm{y}$ in $\mathrm{S}$. If there exists an isomorphism between $\mathrm{S}$ and $\mathrm{S}$ ', then $\mathrm{S}$ and $\mathrm{S}^{\prime}$ are said to be isomorphic, denoted by $\mathrm{S} \approx \mathrm{S}$ '.

Order of an element is cardinality of set generated by that element.

\section{USEFUL THEOREM}

The following theorem about sets, S and S', closed under operations are well known and easy to prove:-

Theorem: - If there exists an isomorphism between $\mathrm{S}$ and S' and the operation on $\mathrm{S}$ is associative then an operation on $\mathrm{S}$ ' is also associative.

\section{OPERATIONS ON A SET WITH 2, 3 AND 4 ELEMENTS}

Number of binary operation on a set of two elements is 16 .

Number of associative binary operation on a set of two elements is 8.See [2].

Number of binary operation on a set of three elements is 19683.

Number of associative binary operation on a set of three elements is 113.See [2] \&[3].

Number of binary operation on a set of four elements is 4,294,967,296.

Number of associative binary operation on a set of four elements is 3492.See [5]

\section{OPERATIONS ON A SET WITH FIVE ELEMENTS}

As mentioned in the introduction, the number of possible binary operations on a set of five elements is $298,023,223,876,953,125$. We now proceed to answer the question: How many binary operations on a set of five elements are associative?

For a five-element set $\mathrm{S}$ proving associatively for a given operation amounts to verify different equations $(x y) z=x(y z)$, where $\mathrm{x}, \mathrm{y}, \mathrm{z}$ are elements of $\mathrm{S}$. A single counterexample suffices to show that a given operation is not associative. Clearly, counterexamples need not to be unique.

Now we discuss alternative ways to check the associativity. Define $\Phi_{\mathrm{a}}$ from $\left(\mathrm{S},{ }^{*}\right)$ into $\left(\mathrm{S},{ }^{*}\right)$, by $\Phi_{\mathrm{a}}(\mathrm{x})=\mathrm{a} * \mathrm{x}$ for all $\mathrm{x}$ in $\mathrm{S}$, where a belong to $\mathrm{S}$. If composition of $\Phi_{\mathrm{a}}$ with $\Phi_{\mathrm{b}}$ is equal to $\Phi_{\mathrm{a} * \mathrm{~b}}$ for all a, b in $\mathrm{S}$ then we say that $\mathrm{S}$ is associatively under the binary operation *. 
Explanation: -

$\mathrm{a} *(\mathrm{~b} * \mathrm{c})=\Phi_{\mathrm{a}}(\mathrm{b} * \mathrm{c})=\Phi_{\mathrm{a}}\left(\Phi_{\mathrm{b}}(\mathrm{c})\right)$

$(\mathrm{a} * \mathrm{~b}) * \mathrm{c}=\Phi_{\mathrm{a} * \mathrm{~b}}(\mathrm{c})$

From (1) and (2) we conclude that above definition is equivalent with definition of associatively of binary operation * on set S. See [3]

\section{ALOGRITHM FOR FINDING NUMBER OF ASSOCIATIVE BINARY OPERATIONON N-ELEMENT}

Algorithm given below is taken from [3], [4] and [5] .The analysis of the associative binary operations on n-element set $\mathrm{S}$ will now divide into 3 steps:-

1). Partition the set of $n^{n}$ mappings in such a way that element of same partition can be obtained by using one-one and onto mapping from $\mathrm{S}$ onto $\mathrm{S}$.

2). Rearrange the partition according to their order of any element of the partition.(Say order as k).

3).Calculate the contribution towards number of associative binary operations when one row is fill by the first element of $\mathrm{i}^{\text {th }}$ partition which can also fill $\mathrm{k}-1$ more rows and remaining row $\mathrm{n}-\mathrm{k}$ can be filled by $\mathrm{i}^{\text {th }}$ and onwards partitions (if any) with two conditions given below.

Conditions: - Before starting calculation, firstly we insured that no associative table counted twice. For this, we make some rules:-

(i) If we fixed $\mathrm{r}^{\text {th }}$ row from any element $\mathrm{i}^{\text {th }}$ partition (which has order $\mathrm{k}$ ) then we can fill at least k-1 more rows. Remaining unfilled rows can by filled with element of $i^{\text {th }}$ and onwards partition (if any) however selected element of $i^{\text {th }}$ partition cannot fill the unfilled rows before selected row.

(ii) If table contains $n$ different entry of $i^{\text {th }}$ partition then contribution towards number of associative operations counted is $1 / \mathrm{n}$.

Explanation (How partition reduces the checking of associative work):- Firstly, we prove that Table-1 and Table-2 given below are isomorphic.

\begin{tabular}{|c|c|c|c|c|c|c|c|c|c|c|c|}
\hline$*_{1}$ & $\mathrm{a}$ & $\mathrm{b}$ & c & $\mathrm{d}$ & e & $*_{2}$ & $\mathrm{a}$ & $\mathrm{b}$ & c & $\mathrm{d}$ & e \\
\hline $\mathrm{a}$ & $\mathrm{e}$ & $\mathrm{a}$ & $\mathrm{a}$ & $\mathrm{c}$ & $b$ & $\mathrm{a}$ & & & & & \\
\hline $\mathrm{b}$ & & & & & & $\mathrm{b}$ & $\mathrm{b}$ & $\mathrm{d}$ & $\mathrm{b}$ & $\mathrm{c}$ & $\mathrm{a}$ \\
\hline $\mathrm{c}$ & & & & & & $\mathrm{c}$ & & & & & \\
\hline $\mathrm{d}$ & & & & & & $d$ & & & & & \\
\hline $\mathrm{e}$ & & & & & & $\mathrm{e}$ & & & & & \\
\hline \multicolumn{6}{|c|}{ Table-1 } & \multicolumn{6}{|c|}{ Table-2 } \\
\hline
\end{tabular}

Here, if we define an isomorphic mapping from $\mathrm{S}$ to $\mathrm{S}$ by $\mathrm{f}$ (a) $=b, f(b)=c, f(c)=a, f(d)=e$ and $f(e)=d$. Then we get

$$
\begin{aligned}
& \mathrm{a} *_{1} a=e \Rightarrow \mathrm{f}(\mathrm{e})=\mathrm{f}(\mathrm{a}) *_{2} \mathrm{f}(\mathrm{a})=>\mathrm{d}=\mathrm{b} *_{2} \mathrm{~b} \\
& \mathrm{a} *_{1} b=a \Rightarrow \mathrm{f}(\mathrm{a})=\mathrm{f}(\mathrm{a}){ }_{2} \mathrm{f}(\mathrm{b})=>\mathrm{b}=\mathrm{b} *_{2} \mathrm{c}
\end{aligned}
$$

$$
\begin{aligned}
& \mathrm{a} *{ }_{1} c=a \Rightarrow \mathrm{f}(\mathrm{a})=\mathrm{f}(\mathrm{a}){ }_{2} \mathrm{f}(\mathrm{c})=>\mathrm{b}=\mathrm{b} *_{2} \mathrm{a} \\
& \mathrm{a} *_{1} d=c=>\mathrm{f}(\mathrm{c})=\mathrm{f}(\mathrm{a}) *_{2} \mathrm{f}(\mathrm{d})=>\mathrm{a}=\mathrm{b} *_{2} \mathrm{e} \\
& \mathrm{a} *_{1} e=b \Rightarrow>\mathrm{f}(\mathrm{b})=\mathrm{f}(\mathrm{a}) *_{2} \mathrm{f}(\mathrm{e})=>\mathrm{c}=\mathrm{b} *{ }_{2} \mathrm{~d}
\end{aligned}
$$

Then composition table after applying above isomorphic mapping to Table-1, we get table-2. Hence, table- 1 and table2 are isomorphic.

Similarly, we can prove that if eaacb comes in second row is isomorphic to bdbca in third row, if eaacb comes in third row is isomorphic to bdbca in first row, if eaacb comes in fourth row is isomorphic to bdbca in fifth row and if eaacb comes in fifth row is isomorphic to bdbca in fouth row. Hence, table with at least one row eaacb is isomorphic to table with at least one row bdbca (row number of bdbca may be different from row number of eaacb).

Hence, there is no need to check associative composition table for bdbca when associative composition table eaacb is checked because they give isomorphic composition table. (By theorem stated in section 3 if a composition table with eaacb is associative then isomorphic composition having row bdbca is also associative).

As above discussion, if we apply any one-one and onto to eaacb we get $\{$ bcaad, bcabd, ........, eebad $\}$ which forms a partition and now our calculation is reduced 120 times (out of 120 elements now we have to check associativity only for single element).

On the basis of above algorithm, now we proceed towards finding number of associative operations on a set with five elements.

\subsection{Step $1^{\text {st }}$}

If set $\mathrm{S}$ has $\mathrm{n}$ elements, then total number of mapping possible from set $S$ to $S=n^{n}$

In our problem $n=5$, then total number of mapping possible from set $\mathrm{S}$ to $\mathrm{S}=3125$.

Here we consider $S=\{0,1,2,3,4\}$ and first digit of each mapping comes from 0 , second comes from 1 , third comes from 2, fourth comes from 3 and last comes from 4.

\begin{tabular}{|r|r|lr|}
\hline $\begin{array}{l}\text { Partition } \\
\text { No }\end{array}$ & $\begin{array}{l}\text { First element of } \\
\text { Partition }\end{array}$ & $\begin{array}{l}\text { Total number of } \\
\text { element } \\
\text { partition }\end{array}$ & $\begin{array}{r}\text { in } \\
\hline 1\end{array}$ \\
\hline 2 & 00000 & 60 \\
\hline 3 & 00001 & 20 \\
\hline 4 & 00011 & 60 \\
\hline 5 & 00012 & 60 \\
\hline 6 & 00013 & 120 \\
\hline 7 & 00014 & 60 \\
\hline 8 & 00033 & 30 \\
\hline 9 & 00034 & 30 \\
\hline 10 & 00043 & 20 \\
\hline 11 & 00111 & 120 \\
\hline 12 & 00112 & \\
\hline
\end{tabular}




\begin{tabular}{|c|c|c|}
\hline 13 & 00114 & 60 \\
\hline 14 & 00122 & 60 \\
\hline 15 & 00123 & 120 \\
\hline 16 & 00124 & 120 \\
\hline 17 & 00133 & 120 \\
\hline 18 & 00134 & 60 \\
\hline 19 & 00143 & 60 \\
\hline 20 & 00224 & 60 \\
\hline 21 & 00234 & 20 \\
\hline 22 & 00243 & 60 \\
\hline 23 & 00322 & 120 \\
\hline 24 & 00342 & 40 \\
\hline 25 & 01234 & 1 \\
\hline 26 & 01243 & 10 \\
\hline 27 & 01322 & 60 \\
\hline 28 & 01342 & 20 \\
\hline 29 & 02111 & 60 \\
\hline 30 & 02112 & 60 \\
\hline 31 & 02113 & 120 \\
\hline 32 & 02143 & 15 \\
\hline 33 & 02311 & 120 \\
\hline 34 & 02341 & 30 \\
\hline 35 & 10000 & 20 \\
\hline 36 & 10001 & 60 \\
\hline 37 & 10002 & 120 \\
\hline 38 & 10012 & 120 \\
\hline 39 & 10022 & 60 \\
\hline 40 & 10023 & 120 \\
\hline 41 & 10043 & 60 \\
\hline 42 & 10342 & 20 \\
\hline 43 & 12000 & 60 \\
\hline 44 & 12001 & 120 \\
\hline 45 & 12003 & 120 \\
\hline 46 & 12300 & 120 \\
\hline 47 & 12340 & 24 \\
\hline
\end{tabular}

\subsection{Step $2^{\text {nd }}$}

\begin{tabular}{|l|l|c|c|}
\hline $\begin{array}{l}\text { Partition } \\
\text { No }\end{array}$ & $\begin{array}{l}\text { First } \\
\text { element } \\
\text { of } \\
\text { Partition }\end{array}$ & $\begin{array}{l}\text { Order of an } \\
\text { element of } \\
\text { partition }\end{array}$ & $\begin{array}{c}\text { Generated } \\
\text { elements belongs } \\
\text { to partition }\end{array}$ \\
\hline
\end{tabular}

\begin{tabular}{|c|c|c|c|}
\hline 1 & 10342 & 6 & $1,21,40,21,1,47$ \\
\hline 2 & 12340 & 5 & $2,2,2,2,47$ \\
\hline 3 & 00123 & 4 & $3,27,25,46$ \\
\hline 4 & 10023 & 4 & $4,23,34,41$ \\
\hline 5 & 12003 & 4 & $5,15,42,15$ \\
\hline 6 & 12300 & 4 & $6,35,6,45$ \\
\hline 7 & 02341 & 4 & $7,39,7,47$ \\
\hline 8 & 00013 & 3 & $8,25,46$ \\
\hline 9 & 00112 & 3 & $9,25,46$ \\
\hline 10 & 00124 & 3 & $10,22,44$ \\
\hline 11 & 02113 & 3 & $11,42,33$ \\
\hline 12 & 02311 & 3 & $12,12,45$ \\
\hline 13 & 10002 & 3 & $13,41,34$ \\
\hline 14 & 10012 & 3 & $14,41,34$ \\
\hline 15 & 12001 & 3 & $15,15,42$ \\
\hline 16 & 00122 & 3 & $16,26,46$ \\
\hline 17 & 00143 & 3 & $17,43,36$ \\
\hline 18 & 10022 & 3 & $18,41,34$ \\
\hline 19 & 12000 & 3 & $19,19,43$ \\
\hline 20 & 00342 & 3 & $20,20,45$ \\
\hline 21 & 01342 & 3 & $21,21,47$ \\
\hline 22 & 00014 & 2 & 22,44 \\
\hline 23 & 00133 & 2 & 23,41 \\
\hline 24 & 00322 & 2 & 24,42 \\
\hline 25 & 00001 & 2 & 25,46 \\
\hline 26 & 00011 & 2 & 26,46 \\
\hline 27 & 00012 & 2 & 27,46 \\
\hline 28 & 00114 & 2 & 28,44 \\
\hline 29 & 00134 & 2 & 29,43 \\
\hline 30 & 00243 & 2 & 30,45 \\
\hline 31 & 01322 & 2 & 31,45 \\
\hline 32 & 02111 & 2 & 32,43 \\
\hline 33 & 02112 & 2 & 33,42 \\
\hline 34 & 10001 & 2 & 34,41 \\
\hline 35 & 10043 & 2 & 35,45 \\
\hline 36 & 00043 & 2 & 36,43 \\
\hline 37 & 00111 & 2 & 37,46 \\
\hline 38 & 10000 & 2 & 38,44 \\
\hline 39 & 02143 & 2 & 39,47 \\
\hline 40 & 01243 & 2 & 40,47 \\
\hline 41 & 00033 & 1 & 41 \\
\hline
\end{tabular}




\begin{tabular}{|l|l|l|l|}
\hline 42 & 00224 & 1 & 42 \\
\hline 43 & 00034 & 1 & 43 \\
\hline 44 & 00004 & 1 & 44 \\
\hline 45 & 00234 & 1 & 45 \\
\hline 46 & 00000 & 1 & 46 \\
\hline 47 & 01234 & 1 & 47 \\
\hline
\end{tabular}

\subsection{Step $3^{\text {rd }}$}

\begin{tabular}{|c|c|c|c|}
\hline $\begin{array}{l}\text { Partition- } \\
\text { No }\end{array}$ & $\begin{array}{l}\text { First } \\
\text { element of } \\
\text { Partition }\end{array}$ & $\begin{array}{l}\text { Assumed } \\
\text { row }\end{array}$ & $\begin{array}{l}\text { Contribution } \\
\text { towards number } \\
\text { of associative }\end{array}$ \\
\hline \multirow[t]{5}{*}{1} & 10342 & 1 & 0 \\
\hline & 10342 & 2 & 0 \\
\hline & 10342 & 3 & 0 \\
\hline & 10342 & 4 & 0 \\
\hline & 10342 & 5 & 0 \\
\hline \multirow[t]{5}{*}{2} & 12340 & 1 & 6 \\
\hline & 12340 & 2 & 6 \\
\hline & 12340 & 3 & 6 \\
\hline & 12340 & 4 & 6 \\
\hline & 12340 & 5 & 6 \\
\hline \multirow[t]{5}{*}{3} & 00123 & 1 & 0 \\
\hline & 00123 & 2 & 0 \\
\hline & 00123 & 3 & 0 \\
\hline & 00123 & 4 & 120 \\
\hline & 00123 & 5 & 120 \\
\hline \multirow[t]{5}{*}{4} & 10023 & 1 & 0 \\
\hline & 10023 & 2 & 0 \\
\hline & 10023 & 3 & 0 \\
\hline & 10023 & 4 & 120 \\
\hline & 10023 & 5 & 120 \\
\hline \multirow[t]{5}{*}{5} & 12003 & 1 & 0 \\
\hline & 12003 & 2 & 0 \\
\hline & 12003 & 3 & 0 \\
\hline & 12003 & 4 & 240 \\
\hline & 12003 & 5 & 120 \\
\hline \multirow[t]{5}{*}{6} & 12300 & 1 & 120 \\
\hline & 12300 & 2 & 60 \\
\hline & 12300 & 3 & 60 \\
\hline & 12300 & 4 & 60 \\
\hline & 12300 & 5 & 0 \\
\hline
\end{tabular}

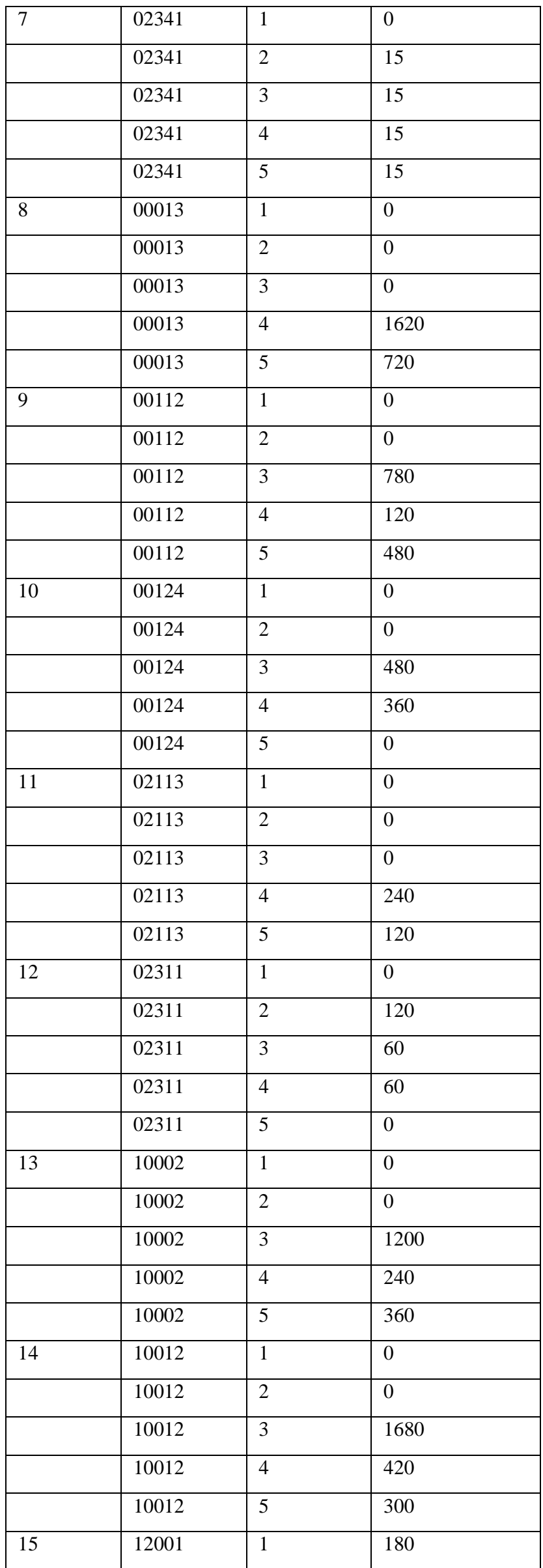




\begin{tabular}{|c|c|c|c|}
\hline & 12001 & 2 & 180 \\
\hline & 12001 & 3 & 60 \\
\hline & 12001 & 4 & 0 \\
\hline & 12001 & 5 & 0 \\
\hline \multirow[t]{5}{*}{16} & 00122 & 1 & 0 \\
\hline & 00122 & 2 & 0 \\
\hline & 00122 & 3 & 480 \\
\hline & 00122 & 4 & 60 \\
\hline & 00122 & 5 & 0 \\
\hline \multirow[t]{5}{*}{17} & 00143 & 1 & 0 \\
\hline & 00143 & 2 & 0 \\
\hline & 00143 & 3 & 0 \\
\hline & 00143 & 4 & 0 \\
\hline & 00143 & 5 & 0 \\
\hline \multirow[t]{5}{*}{18} & 10022 & 1 & 0 \\
\hline & 10022 & 2 & 0 \\
\hline & 10022 & 3 & 1080 \\
\hline & 10022 & 4 & 120 \\
\hline & 10022 & 5 & 60 \\
\hline \multirow[t]{5}{*}{19} & 12000 & 1 & 600 \\
\hline & 12000 & 2 & 30 \\
\hline & 12000 & 3 & 30 \\
\hline & 12000 & 4 & 0 \\
\hline & 12000 & 5 & 0 \\
\hline \multirow[t]{5}{*}{20} & 00342 & 1 & 0 \\
\hline & 00342 & 2 & 0 \\
\hline & 00342 & 3 & 80 \\
\hline & 00342 & 4 & 80 \\
\hline & 00342 & 5 & 80 \\
\hline \multirow[t]{5}{*}{21} & 01342 & 1 & 0 \\
\hline & 01342 & 2 & 0 \\
\hline & 01342 & 3 & 120 \\
\hline & 01342 & 4 & 120 \\
\hline & 01342 & 5 & 120 \\
\hline \multirow[t]{5}{*}{22} & 00014 & 1 & 0 \\
\hline & 00014 & 2 & 9600 \\
\hline & 00014 & 3 & 1380 \\
\hline & 00014 & 4 & 1500 \\
\hline & 00014 & 5 & 0 \\
\hline \multirow[t]{2}{*}{23} & 00133 & 1 & 0 \\
\hline & 00133 & 2 & 5520 \\
\hline
\end{tabular}

\begin{tabular}{|c|c|c|c|}
\hline & 00133 & 3 & 960 \\
\hline & 00133 & 4 & 0 \\
\hline & 00133 & 5 & 1200 \\
\hline \multirow[t]{5}{*}{24} & 00322 & 1 & 0 \\
\hline & 00322 & 2 & 0 \\
\hline & 00322 & 3 & 1680 \\
\hline & 00322 & 4 & 480 \\
\hline & 00322 & 5 & 0 \\
\hline \multirow{5}{*}{25} & 00001 & 1 & 0 \\
\hline & 00001 & 2 & 29680 \\
\hline & 00001 & 3 & 4690 \\
\hline & 00001 & 4 & 4210 \\
\hline & 00001 & 5 & 4720 \\
\hline \multirow[t]{5}{*}{26} & 00011 & 1 & 0 \\
\hline & 00011 & 2 & 15600 \\
\hline & 00011 & 3 & 1300 \\
\hline & 00011 & 4 & 1270 \\
\hline & 00011 & 5 & 970 \\
\hline \multirow[t]{5}{*}{27} & 00012 & 1 & 0 \\
\hline & 00012 & 2 & 660 \\
\hline & 00012 & 3 & 540 \\
\hline & 00012 & 4 & 210 \\
\hline & 00012 & 5 & 150 \\
\hline \multirow[t]{5}{*}{28} & 00114 & 1 & 0 \\
\hline & 00114 & 2 & 4680 \\
\hline & 00114 & 3 & 360 \\
\hline & 00114 & 4 & 180 \\
\hline & 00114 & 5 & 0 \\
\hline \multirow[t]{5}{*}{29} & 00134 & 1 & 0 \\
\hline & 00134 & 2 & 3480 \\
\hline & 00134 & 3 & 1140 \\
\hline & 00134 & 4 & 0 \\
\hline & 00134 & 5 & 0 \\
\hline \multirow[t]{5}{*}{30} & 00243 & 1 & 0 \\
\hline & 00243 & 2 & 0 \\
\hline & 00243 & 3 & 0 \\
\hline & 00243 & 4 & 1560 \\
\hline & 00243 & 5 & 1560 \\
\hline \multirow[t]{3}{*}{31} & 01322 & 1 & 0 \\
\hline & 01322 & 2 & 0 \\
\hline & 01322 & 3 & 1680 \\
\hline
\end{tabular}




\begin{tabular}{|c|c|c|c|}
\hline & 01322 & 4 & 780 \\
\hline & 01322 & 5 & 0 \\
\hline \multirow[t]{5}{*}{32} & 02111 & 1 & 0 \\
\hline & 02111 & 2 & 840 \\
\hline & 02111 & 3 & 60 \\
\hline & 02111 & 4 & 0 \\
\hline & 02111 & 5 & 0 \\
\hline \multirow[t]{5}{*}{33} & 02112 & 1 & 0 \\
\hline & 02112 & 2 & 270 \\
\hline & 02112 & 3 & 270 \\
\hline & 02112 & 4 & 0 \\
\hline & 02112 & 5 & 0 \\
\hline \multirow[t]{5}{*}{34} & 10001 & 1 & 2460 \\
\hline & 10001 & 2 & 780 \\
\hline & 10001 & 3 & 0 \\
\hline & 10001 & 4 & 0 \\
\hline & 10001 & 5 & 0 \\
\hline \multirow[t]{5}{*}{35} & 10043 & 1 & 460 \\
\hline & 10043 & 2 & 200 \\
\hline & 10043 & 3 & 0 \\
\hline & 10043 & 4 & 200 \\
\hline & 10043 & 5 & 200 \\
\hline \multirow[t]{5}{*}{36} & 00043 & 1 & 0 \\
\hline & 00043 & 2 & 0 \\
\hline & 00043 & 3 & 0 \\
\hline & 00043 & 4 & 720 \\
\hline & 00043 & 5 & 720 \\
\hline \multirow[t]{5}{*}{37} & 00111 & 1 & 0 \\
\hline & 00111 & 2 & 4160 \\
\hline & 00111 & 3 & 80 \\
\hline & 00111 & 4 & 40 \\
\hline & 00111 & 5 & 20 \\
\hline \multirow[t]{5}{*}{38} & 10000 & 1 & 3440 \\
\hline & 10000 & 2 & 20 \\
\hline & 10000 & 3 & 0 \\
\hline & 10000 & 4 & 0 \\
\hline & 10000 & 5 & 0 \\
\hline \multirow[t]{4}{*}{39} & 02143 & 1 & 0 \\
\hline & 02143 & 2 & 80 \\
\hline & 02143 & 3 & 80 \\
\hline & 02143 & 4 & 50 \\
\hline
\end{tabular}

\begin{tabular}{|c|c|c|c|}
\hline & 02143 & 5 & 50 \\
\hline \multirow[t]{5}{*}{40} & 01243 & 1 & 0 \\
\hline & 01243 & 2 & 0 \\
\hline & 01243 & 3 & 0 \\
\hline & 01243 & 4 & 1190 \\
\hline & 01243 & 5 & 1190 \\
\hline \multirow[t]{5}{*}{41} & 00033 & 1 & 12810 \\
\hline & 00033 & 2 & 0 \\
\hline & 00033 & 3 & 0 \\
\hline & 00033 & 4 & 8735 \\
\hline & 00033 & 5 & 0 \\
\hline \multirow[t]{5}{*}{42} & 00224 & 1 & 5730 \\
\hline & 00033 & 2 & 0 \\
\hline & 00033 & 3 & 4290 \\
\hline & 00033 & 4 & 0 \\
\hline & 00033 & 5 & 1470 \\
\hline \multirow[t]{5}{*}{43} & 00034 & 1 & 5870 \\
\hline & 00034 & 2 & 0 \\
\hline & 00034 & 3 & 4290 \\
\hline & 00034 & 4 & 0 \\
\hline & 00034 & 5 & 1470 \\
\hline \multirow[t]{5}{*}{44} & 00004 & 1 & 3205 \\
\hline & 00004 & 2 & 0 \\
\hline & 00004 & 3 & 0 \\
\hline & 00004 & 4 & 0 \\
\hline & 00004 & 5 & 1425 \\
\hline \multirow[t]{5}{*}{45} & 00234 & 1 & 1585 \\
\hline & 00234 & 2 & 0 \\
\hline & 00234 & 3 & 680 \\
\hline & 00234 & 4 & 530 \\
\hline & 00234 & 5 & 420 \\
\hline \multirow[t]{5}{*}{46} & 00000 & 1 & 536 \\
\hline & 00000 & 2 & 0 \\
\hline & 00000 & 3 & 0 \\
\hline & 00000 & 4 & 0 \\
\hline & 00000 & 5 & 0 \\
\hline \multirow[t]{5}{*}{47} & 01234 & 1 & 1 \\
\hline & 01234 & 2 & 0 \\
\hline & 01234 & 3 & 0 \\
\hline & 01234 & 4 & 0 \\
\hline & 01234 & 5 & 0 \\
\hline
\end{tabular}


Summary of Section

\begin{tabular}{|c|c|}
\hline Partition-No & $\begin{array}{l}\text { Total contribution towards number of } \\
\text { associative binary operations of partition }\end{array}$ \\
\hline 1 & 0 \\
\hline 2 & 30 \\
\hline 3 & 240 \\
\hline 4 & 240 \\
\hline 5 & 360 \\
\hline 6 & 300 \\
\hline 7 & 60 \\
\hline 8 & 2340 \\
\hline 9 & 1380 \\
\hline 10 & 840 \\
\hline 11 & 360 \\
\hline 12 & 240 \\
\hline 13 & 1800 \\
\hline 14 & 2400 \\
\hline 15 & 420 \\
\hline 16 & 540 \\
\hline 17 & 0 \\
\hline 18 & 1260 \\
\hline 19 & 660 \\
\hline 20 & 240 \\
\hline 21 & 360 \\
\hline 22 & 12480 \\
\hline 23 & 7680 \\
\hline 24 & 2160 \\
\hline 25 & 43300 \\
\hline 26 & 18600 \\
\hline 27 & 1560 \\
\hline 28 & 5220 \\
\hline 29 & 4620 \\
\hline 30 & 3120 \\
\hline 31 & 2460 \\
\hline 32 & 900 \\
\hline 33 & 540 \\
\hline 34 & 3240 \\
\hline 35 & 1060 \\
\hline 36 & 1440 \\
\hline 37 & 4300 \\
\hline
\end{tabular}

\begin{tabular}{|r|r|}
\hline 38 & 3460 \\
\hline 39 & 260 \\
\hline 40 & 2380 \\
\hline 41 & 21545 \\
\hline 42 & 11490 \\
\hline 43 & 9465 \\
\hline 44 & 4630 \\
\hline 45 & 3215 \\
\hline 46 & 536 \\
\hline 47 & 1 \\
\hline Total & 183732 \\
\hline
\end{tabular}

\section{CONCLUSIONS}

The conclusion of this paper is that among the $\mathbf{2 9 8 , 0 2 3 , 2 2 3 , 8 7 6 , 9 5 3 , 1 2 5}$ different operations on a Fiveelement set, $S=\{0,1,2,3,4\}$.

\section{FUTURE WORK}

One can find out Associative binary Operations on a nElement Set by using same Algorithm , which we have used for Five-element and also verify the one of the result for six element set is $\mathbf{1 7 0 6 1 1 1 8}$ [7].

\section{REFERENCES}

[1] Man-Keung Siu, Which Latin Squares are Cayley Tables?, AMS Monthly, 98 (1991) 625-627

[2] Friorik Diego and Kristǐn Halla Jǒnsdǒttiǐ , Associative Operations on a Three-Element Set, TMME, vol 5, nos2 \&3 , pp 257-268, 2008

[3] Amit Sehgal and ManMohan , Associative Binary operation on a set with Three elements, International Journal of Essential Sciences, Vol 5, No. 1, pp1-8,2011 (ISSN:0973-8436)

[4] Sarita and Amit Sehgal, Using Partition Calculation for number of associative operation is reduced. Proceeding of International Conference on Mathematics and Soft Computing (Application in Engineering) pp437-446 Dec $4-5,2010$

[5] Amit Sehgal, Sarita and Sunil Dua, Associative Binary operation on a set with Four elements, International Journal of Computer Science and Communication Engineering Volume 1 Issue 2 paper id IJCSCE 121204, 2013 (ISSN:2319-7080)

[6] George E. Forsythe, Swac Computers 126 distinct semi groups of order 4 Proc. Amer. Math. Soc. 6 (1955), 443447.

[7] Lyle Ramshaw, The On-Line Encyclopedia of Integer Sequences -A023814 (Number of associative binary operations on an n-set

[8] A.Distler and T. Kelsey, The semigroups of order 9 and their automorphism groups, arXiv preprint arXiv: $1301.6023,2013$ 